\section{Spinal subdural haematoma in a parturient after attempted epidural anaesthesia}

\section{To the Editor:}

I was extremely interested to read the case report of "Spinal subdural haematoma in a parturient after attempted epidural anaesthesia"' and was impressed by the speed of diagnosis and subsequent management.

The finding of an elevated alkaline phosphatase in association with normal liver enzymes may indeed be due to biliary cholestasis (intra- or extrahepatic) but of course it is not the only cause. ${ }^{2}$ Bone pathology may give rise to an elevated alkaline phosphatase (e.g., osteomalacia, primary-hyperparathyroidism). In this case the patient had experienced "backache" for six hours before admission (although this pain had resolved before transfer from another hospital). Was there any history of back trauma and had the patient taken any analgesic drugs (such as NSAIDs)? In addition, alkaline phosphatase may also be elevated in the last trimester of pregnancy.

The aetiology of the subdural haematoma in this complicated case was not clearly established and it is a pity that the patient was lost to further follow-up and investigation.

\section{S. Jankowski MRCP FRCA \\ Department of Intensive Care \\ Erasme Hospital \\ Route de Lennik 808 \\ Bruxelles B-1070 \\ Belgium}

\section{REFERENCES}

1 Lao TT, Halpern SH, MacDonald D, Huh C. Spinal subdural haematoma in a parturient after attempted epidural anaesthesia. Can J Anaesth 1993; 40: 340-5.

2 Zilva JP, Pannall PR. Clinical Chemistry in Diagnosis and Treatment 3rd ed. London: Lloyd-Luke, 1979.

\section{$R E P L Y$}

The aetiology of the elevated alkaline phosphatase (approximately seven times normal) in this case remains unknown. She had no history of musculoskeletal trauma and was not taking any non-steroidal antiinflammatory drugs.

The alkaline phosphatase may be elevated for a number of reasons. In this case, we had not pursued the diagnosis because we assumed that it was due to the pregnancy or pre-eclampsia. However, elevation of the enzyme to more than four times normal is unusual for normal pregnancy. Isolated pre-eclampsia (without HELLP syndrome) does not increase the alkaline phosphatase further, although more patients may hove elevations within the normal pregnancy range. ${ }^{l}$

Bone disease may also increase the alkaline phosphatase. It is usually possible to distinguish between the enzyme produced by bone and that by liver. In addition, an elevated 5: nucleotidase would point to liver for the origin of the enzyme. ${ }^{2}$ Unfortunately, we did not do confirmatory tests.

Finally, as Dr. Elliott points out in his commentary, phenothiazines may have been the cause.

In summary, without benefit of additional tests, it is most likely that the elevated alkaline phosphatase ws increased because of a combination of pregnancy and drug use. There was no clinical evidence for bone disease or trauma although a process as hyperparathyroidism cannot be ruled out.

S. Halpern MD FRCPC

Women's College Hospital

Toronto

REFERENCES

1 Elhad H, Gleicher N. Physiologic changes in normal pregnancy. In: Gleicher N (Ed.). Principles of Medical Therapy in Pregnancy. Plenum Medical Book Co. 1985; 33-56.

2 Podolsky DK, Isselbacher KJ. Diagnostic tests in liver disease. In: Wilson JD et al. (Eds.). Harrison's Principles of Internal Medicine 12th ed. McGraw Hill; New York, 1991; 1308-11.

\section{Accidental total spinal}

To the Editor:

We read with interest the article by Palkar et al. ${ }^{1}$ and would like to raise a number of points.

First, we wondered why the authors exposed an obese obstetric patient to the risks of general anaesthesia when sensory analgesia was above $T_{4}$ ? We agree that sensory analgesia due to $45 \mathrm{mg}$ lidocaine may not have lasted the duration of surgery, especially as the authors suggested that the analgesia was regressing rapidly. However, this patient had an epidural catheter in situ which was thought to be in the subarachnoid space and, therefore, there was no reason why adequate sensory analgesia could not have been maintained by injecting small increments of local anaesthetic through the catheter: continuous spinal anaesthesia is becoming increasingly popular in obstetric practice.

Second, why did the authors not aspirate the epidural catheter after the positive test dose? The epidural catheter could have been placed in the subdural space which would have resulted in a negative aspiration test. Injection of the test dose could then have produced a tear in the arachnoid mater, and, thereby, resulted in a positive test dose. Aspiration of the catheter at this point in time would then have resulted in a positive aspiration test. However, the authors concluded that the above situation was not 